\title{
Sistema hospitalar como fonte de informações para estimar a mortalidade neonatal e a natimortalidade
}

The Brazilian hospital system as a source of information to estimate stillbirth and neonatal mortality rates

Joyce MA Schramma e Célia L Szwarcwald ${ }^{b}$

a Escola Nacional de Saúde Pública da Fundação Oswaldo Cruz (Fiocruz). Rio de Janeiro, RJ, Brasil. ${ }^{b}$ Departamento de Informações em Saúde do Centro de Informações Científicas e Tecnológicas da Fiocruz. Rio de Janeiro, RJ, Brasil 


\section{Sistema hospitalar como fonte de} informações para estimar a mortalidade neonatal e a natimortalidade The Brazilian hospital system as a source of information to estimate stillbirth and neonatal mortality rates

\section{Joyce MA Schramma e Célia L Szwarcwald}

aEscola Nacional de Saúde Pública da Fundação O swaldo Cruz (Fiocruz). Rio de Janeiro, RJ, Brasil.

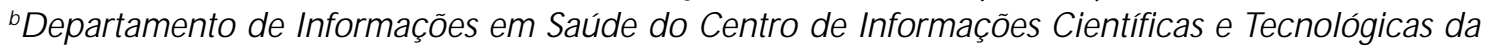
Fiocruz. Rio de Janeiro, RJ, Brasil

\section{Descritores}

Sistemas de informação hospitalar. Mortalidade neonatal, saúde pública\#. Mortalidade fetal\#. Registros de mortalidade\#.

\section{Keywords}

Hospital information systems ${ }^{\#}$.

Neonatal mortality, public health ${ }^{\#}$.

Fetal mortality". Mortality registries ${ }^{\#}$.

\section{Resumo}

\section{Objetivo}

Apesar da reconhecida importância em acompanhar a evolução temporal da mortalidade infantil precoce, a deficiência das estatísticas vitais no Brasil ainda permanece na agenda atual dos problemas que impedem o seu acompanhamento espaço-temporal. Realizouse estudo com o objetivo de investigar o Sistema de Informações Hospitalares (SIH/SUS) como fonte de informações, para estimar a natimortalidade e a mortalidade neonatal.

\section{Métodos}

Propõe-se um método para estimar a natimortalidade e a mortalidade neonatal, o qual foi aplicado para todos os Estados das regiões Nordeste, Sul e Sudeste e para o Pará, no ano de 1995. Para fins comparativos, o Sistema de Informações sobre Mortalidade (SIM/MS) foi utilizado para estimar as taxas sob estudo, após a correção do número de nascidos vivos por um método demográfico.

\section{Resultados}

O SIH/SUS forneceu mais óbitos fetais e neonatais precoces do que o SIM/MS em grande parte das unidades federadas da região Nordeste. Adicionalmente para os Estados localizados nas regiões Sul e Sudeste, que apresentam, em geral, boa cobertura do registro de óbitos, as taxas calculadas pelos dois sistemas de informação tiveram valores semelhantes. Conclusões

Considerando a cobertura incompleta das estatísticas vitais no Brasil e a agilidade do SIH/SUS em disponibilizar as informações em meio magnético, conclui-se que o uso do SIH/SUS poderá trazer inúmeras contribuições para análise do comportamento espaço-temporal do componente neonatal da mortalidade infantil no território brasileiro, em anos recentes.
Abstract
Objective
Studies on the evolution of infant mortality rate are very relevant. Nevertheless, lack of vital statistics in Brazil limits the temporal and spatial analysis of this indicator. This study aims to investigate the possible use of the Brazilian Hospital Information System as an alternative information source for stillbirth and neonatal mortality rates by age group. 


\begin{abstract}
Methods
A new method to estimate the stillbirth and neonatal mortality rates is proposed. It was applied in a set of selected Brazilian states in the year of 1995. For comparative purposes, the Brazilian Death Information System was assessed to estimate the mortality rates under study, after adjusting the registered number of live births by using a demographic tool.

Results

By assessing the Hospital Information System a larger number of fetal and early neonatal deaths were observed when compared to data given by the death information system of the Northeastern states. Besides, in the Southern and Southeastern states, where death records are more thorough, the mortality rates calculated using both information sources were very similar.

\section{Conclusions}

The results suggest that the proposed methodology could greatly contribute to the analysis of the spatial-time evolution of stillbirth and neonatal death rates in recent years in Brazil, as data on death registration in the majority of the Brazilian states are less thorough than those from the hospital information system.
\end{abstract}

\section{INTRODUÇÃO}

O comportamento evolutivo nos últimos anos dos componentes da mortalidade infantil, a mortalidade neonatal e a mortalidade pós-neonatal no Brasil foi descrito por diversos autores. Ainda que a tendência geral seja de decréscimo, o estudo de Simões \& Monteiro $^{12}$ mostrou que o declive gradual não vem se dando de forma homogênea no País, refletindo os distintos processos de desenvolvimento econômico e social das regiões brasileiras.

Becker $^{1}$ apontou que o fator explicativo mais importante das tendências de decréscimo que ocorreram no País foi o número de mortes no período pós-neonatal. Entretanto, se a velocidade de declive das mortes no período tardio foi compatível a de outros países, o componente neonatal não mostrou a mesma evolução. Em uma investigação sobre tendência temporal da mortalidade infantil, Leal \& Szwarcwald ${ }^{8}$ concluíram que os níveis de mortalidade neonatal precoce ainda permanecem muito elevados no Estado do Rio de Janeiro, sendo que quanto maior a proximidade do parto, menor é a redução do coeficiente de mortalidade infantil (CMI). Achados semelhantes foram encontrados para outros Estados, como São Paulo e Rio Grande do Sul. ${ }^{13}$

Apesar da reconhecida importância em acompanhar a evolução temporal da mortalidade infantil precoce, tida como um evento-sentinela da qualidade da atenção médica, a deficiência das estatísticas vitais no Brasil ainda permanece na agenda atual como um dos problemas que impedem o acompanhamento espaçotemporal. O sub-registro de óbitos e o atraso do registro ou subenumeração dos nascimentos são apontados como os principais fatores que impedem a estimativa do CMI e seus componentes.
Evidências do sub-registro de nascidos vivos no registro civil e/ou de atraso na notificação foram constatadas no trabalho de Camarano. ${ }^{3}$ Para corrigir o número registrado de nascidos vivos, têm sido utilizados procedimentos diversos, sejam modelos desenvolvidos no País ${ }^{2}$ ou métodos propostos na literatura especializada ${ }^{14}$ que fazem uso de fontes alternativas, como os censos e as Pesquisas Nacionais por Amostragem de Domicílio (PNAD).

Recentemente, o Ministério da Saúde desenvolveu o Sistema de Nascidos Vivos (SINASC). Oficialmente estabelecido no País a partir de 1990, esse sistema visa a melhorar a qualidade da informação sobre nascidos vivos e traçar o perfil epidemiológico de nascimentos. Embora já esteja sendo utilizado como fonte de informações de nascimentos em algumas áreas específicas, o sistema ainda apresenta problemas de cobertura no nível nacional. ${ }^{9}$ Do total de nascidos vivos no Brasil, estimado pela Fundação Instituto de Geografia e Estatística (FIBGE) para o ano de 1995, apenas $82 \%$ foram registrados no SINASC.

Uma outra limitação relevante é o sub-registro de óbitos em diversos Estados. Estima-se que a cobertura do Sistema de Informação sobre Mortalidade do Ministério da Saúde (SIM/MS) seja de aproximadamente $75 \%$. A subnotificação dos óbitos é diferenciada por região, sendo maior nas regiões Norte e Nordeste.

Tendo em vista todas essas questões, é de fundamental importância o estudo de outras bases nacionais de dados que possibilitem acompanhar o comportamento evolutivo da mortalidade infantil. O Sistema de Informações Hospitalares do Sistema Único de Saúde (SIH/SUS), concebido no início da década de 80, é a única fonte de informações de morbidade hospi- 
talar nacional, estimando-se sua cobertura em aproximadamente $70 \%$ do total de internações do País.

O presente trabalho propõe-se a investigar o $\mathrm{SIH} /$ SUS como fonte alternativa de informações para a estimativa da natimortalidade e da mortalidade neonatal hospitalar, apresentando-se uma metodologia para a construção desses coeficientes.

\section{MÉTODOS}

\section{Sobre a Autorização de Internação Hospitalar}

O formulário AIH (Autorização de Internação Hospitalar) é o documento central do sistema. É preenchido cada vez que é caracterizada uma internação, mesmo em casos de transferências entre clínicas de especialidades diferentes dentro de uma mesma unidade hospitalar, ou em casos de transferências para outras unidades hospitalares. Assim, a AIH individualiza o paciente nos casos de internação hospitalar e é o instrumento de informações e cobrança dos serviços prestados aos usuários do SUS. Os dados são processados pelo Datasus, compactados e disponibilizados em CDRom e denominados, respectivamente, de AIH-reduzida e AIH mês a mês, de acordo com o tipo de arquivo e o volume de variáveis incluídas.

No que se refere ao objeto do presente trabalho, algumas especificações do SIH/SUS precisam ser apontadas. O manual do SIH/SUS preconiza o seguinte para as gestantes: as AIH 1 são emitidas a partir do sexto mês de gestação e possuem validade até o parto; o limite máximo para o tempo de permanência nos casos da realização dos procedimentos parto normal e parto cesárea é de 6 dias; se as crianças nascem mortas, os óbitos são registrados na AIH da mãe no campo correspondente ao número de natimortos.

Já em relação aos recém-natos, é estabelecido que não sejam emitidas AIH caso as crianças sejam normais ou possuam uma das três características: (1) filho de mãe diabética, (2) icterícia do recém-nascido ou (3) ser um bebê potencialmente infectado. Se em uma dessas situações o recém-nascido morre, esse óbito também é registrado na AIH da mãe, no campo que especifica a condição de saída da criança. $\mathrm{O}$ atestado de óbito para essa criança é então preenchido, sem que haja emissão de AIH. Uma AIH é emitida para o récem-nato se for detectada uma patologia que necessite de internação e que não esteja incluída nas possibilidades mencionadas anteriormente. ${ }^{10}$

\section{Procedimentos}

Durante o ano de 1995, foram consideradas todas as AIH emitidas para as mulheres cujos campos correspondentes ao número de nascidos vivos e/ou número de nascidos mortos estivessem preenchidos, aqui denominadas de "AIH das mães", e todas as AIH emitidas para as crianças menores de 1 ano, que constituíram as chamadas "AIH das crianças".

Entre as crianças para as quais foram emitidas AIH, elaborou-se um algoritmo que estima, em dias, a idade da criança ao morrer, pela diferença entre a data de saída e a data de internação. Os dias de vida da criança não correspondem à idade fornecida pelo Datasus, já que essa se refere à idade da criança no momento da internação.

Para a construção do numerador das taxas de mortalidade neonatal foram computados, primeiramente, todos os óbitos ocorridos e registrados nas "AIH das mães". Para os casos de duplo registro, envolvendo as variáveis "natimorto" e "saída óbito", prevaleceu a classificação "natimorto". Os óbitos registrados nas "AIH das mães" foram somados aos óbitos contidos nas "AIH das crianças" após o cálculo da idade, compondo, assim, a totalidade de óbitos neonatais precoces e neonatais tardios. O numerador da taxa de natimortalidade foi obtido por meio do campo da AIH "número de nascidos mortos", que inclui todas as mortes fetais.

Para o cálculo do denominador, sob a suposição de que os óbitos no período neonatal captados no sistema SUS correspondem a filhos de gestantes que se internaram em um hospital do SUS para o parto, foram selecionadas todas as "AIH das mães" cujo campo correspondente ao número de nascidos vivos estivesse preenchido. O número total de nascidos vivos declarado nessas AIH foi tomado como o denominador das taxas.

Dessa forma, o próprio sistema forneceu o número de nascidos vivos, o número de nascidos mortos e o número de óbitos entre os nascidos vivos no período neonatal, permitindo calcular as taxas de mortalidade neonatal precoce, neonatal tardia e natimortalidade.

Para o cálculo dessas taxas, elegeu-se um conjunto de unidades federadas, tentando abranger Estados com boa cobertura das estatísticas vitais, como São Paulo, Minas Gerais, Rio de Janeiro e Rio Grande do Sul, e Estados com reconhecida deficiência, como os Estados da região Nordeste. Foi selecionado o ano de 1995, último ano com informações de mortalidade dis- 
poníveis em nível nacional pelo SIM/MS, sendo utilizado a título de comparação para as taxas construídas pelas informações do SIH/SUS.

As taxas de mortalidade neonatal hospitalares, construídas a partir do SIM/MS, tiveram como numerador o número de óbitos em crianças de 0-27 dias ocorrido em hospitais. A estimativa do número de nascidos vivos, para o ano de 1995, foi realizada em duas etapas. Na primeira, corrigiu-se o número de nascidos vivos, declarado no censo de 1991, pelo método de Brass. ${ }^{14}$ Comparando-se esse último com o número de nascimentos registrados no mesmo ano, obteve-se um fator de correção de registro de nascimento para cada unidade federal selecionada. $\mathrm{O}$ fator foi utilizado para corrigir o número de nascidos vivos registrados nos anos de 1992, 1993 e 1994. A segunda etapa consistiu na estimação dos nascidos vivos para o ano de 1995, a partir das estimativas obtidas para os anos anteriores, sob a suposição de crescimento geométrico.

A taxa de natimortalidade, construída a partir do SIM, incluiu no numerador tanto os nascidos mortos quanto as mortes fetais, para que se pudesse garantir a comparação entre as taxas calculadas sob os registros do SIM e do SIH/SUS.

\section{RESULTADOS}

A Tabela 1 permite acompanhar, de forma resumida, a metodologia utilizada para a correção do núme- ro de nascidos vivos em 1995. Destacam-se os fatores de correção obtidos para a região Nordeste, que alcançaram valores muito elevados, como os encontrados para os Estados do Maranhão e do Piauí, de 7,3 e 5,8, respectivamente. Por outro lado, os fatores de ajuste correspondentes aos Estados das regiões Sudeste e Sul foram todos menores que 1,5, sendo que o de Minas Gerais apresentou o menor índice $(1,06)$ entre as unidades federadas estudadas. A cobertura dos nascimentos pelo SIH/SUS apresentou grande amplitude de variação, embora a maioria dos Estados tenha mostrado cobertura próxima a $60 \%$. Destacaram-se Alagoas e Maranhão, com os percentuais mais baixos, de $45 \%$ e $46 \%$, respectivamente, e Minas Gerais e Espírito Santo com os valores mais altos, de $95 \%$ e $81 \%$, respectivamente.

A comparação entre o número de nascidos vivos estimado pelo método de Brass e o número de nascidos vivos obtido pelas informações do SIH/SUS e do SINASC apontam igualmente para uma baixa cobertura do SINASC em uma parte representativa das unidades federadas. Percebe-se, inclusive, que o número de nascidos vivos registrado no SINASC é menor que o número obtido pelo SIH/SUS em alguns Estados da região Nordeste (Tabela 1).

Na Tabela 2 estão dispostos o número de nascidos mortos e o número de óbitos neonatais precoces e tardios registrados nos dois sistemas de informações, SIH/SUS e SIM/MS. Para cada grupo etário foram calculadas as proporções de óbitos registrados no SIH/SUS referentes ao total de mortes hospitalares

Tabela 1 - Correção do número de nascidos vivos em Unidades da Federação selecionadas. Brasil, 1995.

\begin{tabular}{|c|c|c|c|c|c|c|c|c|}
\hline $\begin{array}{l}\text { Unidades } \\
\text { Federais }\end{array}$ & $\begin{array}{l}N^{o} \text { de } \\
\text { NV Censo } \\
1991^{a}\end{array}$ & $\begin{array}{c}N^{\circ} \\
\text { corrigido } \\
\text { de NV }\end{array}$ & $\begin{array}{c}\mathrm{N}^{\circ} \\
\text { registrado } \\
\text { na ERC } \\
1991^{\mathrm{b}}\end{array}$ & $\begin{array}{l}\text { Fator de } \\
\text { correção }\end{array}$ & $\begin{array}{c}\mathrm{N}^{\circ} \\
\text { estimado } \\
\text { de NV } \\
1995\end{array}$ & $\begin{array}{c}\mathrm{N}^{\circ} \\
\text { de NV } \\
\text { SIH/SUS } \\
1995\end{array}$ & $\begin{array}{c}\text { Cobertura }(\%) \\
\text { do no } \\
\text { de NV pelo } \\
\text { SIH/SUS }\end{array}$ & $\begin{array}{r}\mathrm{N}^{\circ} \\
\text { de NV } \\
\text { SINASC } \\
1995^{\mathrm{d}}\end{array}$ \\
\hline Pará - PA & 113.301 & 156.718 & 39.755 & 3,94 & 170.246 & 107.307 & 63 & 96.159 \\
\hline Maranhão - MA & 135.257 & 185.532 & 25.407 & 7,30 & 219.476 & 101.668 & 46 & 56.387 \\
\hline Piauí - PI & 62.065 & 92.663 & 15.846 & 5,85 & 104.193 & 59.593 & 57 & 38.209 \\
\hline Ceará - CE & 150.368 & 218.304 & 71.138 & 3,07 & 263.287 & 161.194 & 61 & 119.228 \\
\hline Rio Gde do Norte - RN & 53.670 & 82.029 & 26.025 & 3,15 & 106.831 & 58.188 & 55 & 59.886 \\
\hline Paraíba - PB & 74.354 & 109.367 & 48.068 & 2,28 & 119.611 & 62.479 & 53 & 26.642 \\
\hline Pernambuco - PE & 160.336 & 237.650 & 86.088 & 2,76 & 279.568 & 148.293 & 53 & 161.290 \\
\hline Alagoas - AL & 63.967 & 100.972 & 26.732 & 3,78 & 131.137 & 59.591 & 45 & 66.642 \\
\hline Sergipe - SE & 36.313 & 57.542 & 23.274 & 2,47 & 74.459 & 36.740 & 49 & 40.316 \\
\hline Bahia - BA & 243.954 & 370.591 & 163.712 & 2,26 & 467.895 & 228.397 & 49 & 163.494 \\
\hline Espírito Santo - ES & 54.348 & 60.962 & 45.529 & 1,34 & 60.597 & 48.740 & 81 & 59.790 \\
\hline Minas Gerais - MG & 233.531 & 311.063 & 293.795 & 1,06 & 308.970 & 292.385 & 95 & 122.796 \\
\hline Rio de Janeiro - RJ & 217.368 & 270.949 & 207.017 & 1,31 & 279.288 & 197.967 & 71 & 266.463 \\
\hline São Paulo - SP & 567.993 & 736.005 & 614.838 & 1,20 & 777.265 & 423.489 & 55 & 691.364 \\
\hline Paraná - PA & 183.276 & 245.755 & 167.404 & 1,47 & 256.273 & 161.161 & 63 & 195.251 \\
\hline Santa Catarina - SC & 98.088 & 123.189 & 93.118 & 1,32 & 121.905 & 80.546 & 67 & 97.969 \\
\hline Rio Gde do Sul - RS & 175.230 & 205.860 & 160.272 & 1,28 & 203.822 & 126.001 & 62 & 188.350 \\
\hline
\end{tabular}

Fonte:

a) FIBGE, Censo Demográfico, 1991

b) FIBGE, Estatísticas do Registro Civil, 1991, 1992, 1993, 1994. Ministério da Saúde, CD ROM AIH, Mês a Mês, 1995

c) Em relação ao número estimado de nascídos vivos em 1995

d) Datasus, 1995

NV: nascidos vivos

SIH/SUS: Sistema de Informações Hospitalares do Sistema Único de Saúde

SINASC: Sistema de Informações sobre Nascidos Vivos 
do SIM/MS. Os resultados encontrados para os natimortos apontam o SIH/SUS como o sistema que melhor captou os óbitos desse grupo em quase todas as unidades federadas estudadas, com exceção dos Estados do Rio de Janeiro, São Paulo e Paraná. Nos Estados do Maranhão, Paraíba, Alagoas e Bahia, o volume de natimortos registrados no SIH/SUS foi cerca de duas vezes maior.

Comparando-se o número de óbitos neonatais precoces ocorridos e registrados nos dois sistemas, nos Estados do Ceará, Alagoas e Bahia, o sistema SIH/ SUS apresentou mais óbitos neonatais precoces do que o SIM. Nos outros Estados da região Nordeste e no Pará, com exceção do Piauí, Pernambuco e Sergipe, o número de óbitos registrados pelo SIH/SUS foi bastante alto quando comparado com o SIM/MS. Nas regiões Sul e Sudeste o SIH/SUS apresentou coberturas próximas a $60 \%$. O Estado de Santa Catarina, cujo percentual correspondente foi de $99 \%$, indica problemas de sub-registro no SIM/MS.

Em relação ao componente tardio da mortalidade neonatal, verifica-se que o número de óbitos computados no SIH/SUS corresponde a $82 \%$ dos óbitos hospitalares registrados no SIM/MS. Os Estados da região Nordeste, considerados em conjunto, apresentam mais óbitos captados pelo SIH/SUS do que pelo SIM/MS, ocorrendo o oposto nas regiões Sul e Sudeste.

As freqüências de óbitos neonatais precoces, categorizadas segundo a fonte de informação ("AIH da mãe" ou "AIH da criança"), mostram que a proporção de óbitos registrados nas "AIH das mães" representa cerca de $48 \%$ das mortes registradas pelo sistema SIH/ SUS, no período neonatal precoce, nas unidades federadas estudadas (Tabela 3). Esse valor tão elevado deve ser ressaltado, já que, em princípio, somente as crianças em situações tidas como de não-gravidade permaneceriam incluídas nas "AIH das mães". Os Estados de Minas Gerais, Rio de Janeiro e Santa Catarina apresentaram os mais altos percentuais.

Tabela 3 - Número de óbitos em crianças de 0-6 dias registrados no SIH/SUS segundo a classificação das AIH como "AlH das mães" ou "AlH das crianças" em Estados selecionados. Brasil, 1995.

\begin{tabular}{lrrrrr}
\hline UF & AlH/criança & $\%$ & AlH/mãe & $\%$ & total \\
\hline PA & 464 & 72 & 187 & 28 & 651 \\
MA & 220 & 63 & 128 & 37 & 348 \\
PI & 102 & 67 & 52 & 33 & 154 \\
CE & 920 & 65 & 506 & 35 & 1.426 \\
RN & 210 & 48 & 229 & 52 & 439 \\
PB & 389 & 71 & 160 & 29 & 549 \\
PE & 741 & 66 & 380 & 34 & 1.121 \\
AL & 230 & 48 & 255 & 52 & 485 \\
SE & 162 & 56 & 127 & 44 & 289 \\
BA & 857 & 45 & 1.065 & 55 & 1.922 \\
ES & 195 & 49 & 202 & 51 & 397 \\
MG & 1.164 & 38 & 1.873 & 62 & 3.037 \\
RJ & 730 & 31 & 1.065 & 69 & 1.795 \\
SP & 2.616 & 52 & 2.458 & 48 & 5.074 \\
PR & 806 & 61 & 513 & 39 & 1.319 \\
SC & 306 & 39 & 489 & 61 & 795 \\
RS & 740 & 73 & 268 & 27 & 1.008 \\
\hline Total & 10.852 & 53,2 & 9.957 & 48,9 & 20.809 \\
\hline
\end{tabular}

Fonte: SIH/SUS, 1995

AlH: Autorização de Internação Hospitalar

$\mathrm{Na}$ Tabela 4 encontram-se as estimativas das taxas de natimortalidade, mortalidade neonatal e seus dois componentes. Primeiramente, nota-se a grande diferença entre as taxas de natimortalidade calculadas a partir do sistema SIH/SUS e aquelas calculadas a partir dos óbitos captados pelo SIM/MS. As diferenças atingem níveis importantes, sobretudo nos Estados da região Nordeste, como no caso do Estado do

Tabela 2 - Número de óbitos registrados no SIH/SUS e SIM/MS por faixa etária em Estados selecionados. Brasil, 1995.

\begin{tabular}{|c|c|c|c|c|c|c|c|c|c|}
\hline \multirow[t]{2}{*}{ UF } & \multicolumn{3}{|c|}{ Natimortos } & \multicolumn{3}{|c|}{ 0-6 dias } & \multicolumn{3}{|c|}{$7-27$ dias } \\
\hline & SIH/SUS & SIM/MS & $\%$ & $\mathrm{SIH} / \mathrm{SUS}$ & SIM/MS & $\%$ & $\mathrm{SIH} / \mathrm{SUS}$ & SIM/MS & $\%$ \\
\hline PA & 1.319 & 1.061 & 124,3 & 651 & 881 & 73,9 & 188 & 243 & 77,4 \\
\hline $\mathrm{MA}$ & 2.360 & 752 & 313,8 & 348 & 460 & 75,7 & 95 & 87 & 109,2 \\
\hline PI & 479 & 732 & 65,4 & 154 & 338 & 45,6 & 49 & 45 & 108,9 \\
\hline CE & 1.967 & 1.418 & 138,7 & 1.426 & 972 & 146,7 & 360 & 284 & 126,8 \\
\hline RN & 724 & 484 & 149,6 & 439 & 521 & 84,3 & 107 & 112 & 95,6 \\
\hline PB & 767 & 353 & 217,3 & 549 & 601 & 91,3 & 172 & 116 & 148,3 \\
\hline PE & 2.122 & 2.317 & 91,6 & 1.121 & 1.973 & 56,8 & 374 & 468 & 79,9 \\
\hline$A L$ & 876 & 419 & 209,1 & 485 & 451 & 107,5 & 146 & 119 & 122,7 \\
\hline SE & 808 & 482 & 167,6 & 289 & 819 & 35,3 & 100 & 105 & 95,3 \\
\hline BA & 2.865 & 1.139 & 251,5 & 1.922 & 1.859 & 103,4 & 445 & 364 & 122,3 \\
\hline ES & 990 & 922 & 107,4 & 397 & 626 & 63,4 & 117 & 151 & 77,5 \\
\hline$M G$ & 5.160 & 4.684 & 110,2 & 3.037 & 4.995 & 60,8 & 650 & 799 & 81,4 \\
\hline RJ & 3.571 & 4.663 & 76,6 & 1.795 & 3.506 & 51,2 & 440 & 807 & 54,5 \\
\hline SP & 5.768 & 10.294 & 56,0 & 5.074 & 8.567 & 59,2 & 1.270 & 1.920 & 66,2 \\
\hline PR & 2.079 & 2.185 & 95,1 & 1.319 & 2.007 & 65,7 & 354 & 421 & 84,1 \\
\hline SC & 1.016 & 563 & 180,5 & 795 & 803 & 99,0 & 180 & 174 & 103,4 \\
\hline $\mathrm{RS}$ & 1.236 & 1.872 & 66,0 & 1.008 & 1.466 & 68,8 & 401 & 447 & 89,7 \\
\hline Total & 34.107 & 34.340 & 99,3 & 20.809 & 30.845 & 67,5 & 5.448 & 6.662 & 81,8 \\
\hline
\end{tabular}


Maranhão, cuja taxa calculada pelo SIH/SUS é seis vezes maior. Nos Estados do Rio Grande do Norte, Alagoas, Sergipe e Bahia, as disparidades, embora menores, são também significativas. A região Sudeste, com reconhecido registro de mortalidade de melhor qualidade, apresentou semelhança entre as taxas de natimortalidade calculadas pelos dois sistemas. Nos Estados do Rio de Janeiro e São Paulo, as taxas de natimortalidade são praticamente as mesmas. Para a região Sul, o Estado de Santa Catarina apresentou a maior diferença entre as taxas calculadas pelos dois sistemas.

A análise dos componentes da taxa de mortalidade neonatal mostrou que, em relação ao período de 0-6 dias, com exceção dos Estados do Piauí, Minas Gerais, Espírito Santo e Rio de Janeiro, as taxas calculadas pelo SIH/SUS foram maiores, destacando-se as diferenças encontradas para os Estados da região Nordeste. Por exemplo, a taxa de mortalidade neonatal precoce estimada pelo SIH/SUS para o Ceará foi de $8,9 / 1.000 \mathrm{NV}$, seis vezes maior que a calculada pelo SIM/MS. No entanto, as estimativas para o Maranhão e Piauí permanecem muito baixas, merecendo investigacão mais detalhada. Para as unidades federadas que integram a região Sul, as taxas calculadas pelos dois sistemas de informação são semelhantes nos Estados do Paraná e Rio Grande do Sul, porém em Santa Catarina o coeficiente calculado pelo SIH/SUS foi bem superior.

Como esperado, as taxas de mortalidade do componente neonatal tardio apresentam níveis mais baixos do que o componente neonatal precoce, uma vez que reconhecidamente o maior volume de mortes infantis ocorre nos primeiros 6 dias de vida. Da mesma forma, como nas análises anteriores, as diferenças entre as taxas calculadas pelo sistema SIH/SUS e pelo SIM/
MS são bem maiores para os Estados da região Nordeste. Na região Sudeste as taxas são bastante semelhantes e na região Sul as taxas calculadas pelo SIH/ SUS são ligeiramente superiores.

\section{DISCUSSÃO}

Os comentários iniciais referem-se ao problema do registro dos nascidos vivos no Brasil, tendo em vista os valores elevados dos fatores estimados para corrigir os dados de nascimento do registro civil, sobretudo na região Nordeste.

Comparando-se o número de nascidos vivos computado no SIH/SUS ao número corrigido de nascidos vivos, observam-se coberturas incompletas, devidas, possivelmente, a outros fatores que não o sub-registro. Acredita-se que as coberturas baixas podem ser atribuídas, em parte, à ocorrência ainda freqüiente de partos domiciliares, principalmente no interior das unidades federadas da região Nordeste. Adicionalmente, as coberturas baixas podem ser explicadas pela grande proporção de partos em unidades hospitalares da rede privada, que não têm convênio com o SUS. A análise das informações da Assistência Médico-Sanitária de $1992^{6}$ aponta que, na região Sudeste, a proporção de leitos privados chega a cerca de $46 \%$, enquanto na região Nordeste atinge $38 \%$. Particularmente para o Estado do Rio de Janeiro, o dado de cobertura hospitalar para o setor privado, de cerca de $30 \%,{ }^{7}$ parece ser consistente em relação à cobertura de nascimentos encontrada no presente estudo.

O SIH/SUS mostrou-se um sistema de informações com maior cobertura dos óbitos fetais do que o SIM/ MS. As diferenças encontradas entre o número de nascidos mortos captados pelos dois sistemas de informação confirmam o alto nível de subnotificação dos óbitos fetais no SIM, sobretudo no Nordeste, como

Tabela 4 - Taxas de natimortilidade (por 1.000 nascimentos) e mortalidade neonatal (precoce e tardia)(por 1.000 nascidos vivos) estimadas pelo SIH/SUS e pelo SIM/MS em Estados selecionados. Brasil, 1995.

\begin{tabular}{|c|c|c|c|c|c|c|c|c|}
\hline \multirow[t]{2}{*}{ UF } & \multicolumn{2}{|c|}{ Taxa de Natimortalidade } & \multicolumn{2}{|c|}{ 0-6 Dias } & \multicolumn{2}{|c|}{ 7-27 Dias } & \multicolumn{2}{|c|}{ 0-28 Dias } \\
\hline & $\mathrm{SIH} / \mathrm{SUS}$ & SIM/MS & $\mathrm{SIH} / \mathrm{SUS}$ & SIM/MS & $\mathrm{SIH} / \mathrm{SUS}$ & SIM/MS & SIH/SUS & SIM/MS \\
\hline PA & 12,1 & 6,2 & 6,0 & 5,2 & 1,8 & 1,4 & 7,8 & 6,6 \\
\hline MA & 22,7 & 3,4 & 3,4 & 2,1 & 1,0 & 0,4 & 4,4 & 2,5 \\
\hline $\mathrm{PI}$ & 8,0 & 7,0 & 2,6 & 3,2 & 0,8 & 0,4 & 3,4 & 3,6 \\
\hline CE & 12,1 & 5,4 & 8,9 & 1,2 & 2,3 & 2,5 & 11,0 & 4,8 \\
\hline $\mathrm{RN}$ & 12,3 & 4,5 & 7,6 & 4,9 & 1,9 & 1,1 & 9,4 & 5,9 \\
\hline PB & 12,1 & 2,9 & 8,8 & 4,2 & 2,8 & 1,0 & 11,6 & 5,2 \\
\hline$P E$ & 14,1 & 8,2 & 7,6 & 7,1 & 2,5 & 1,7 & 10,1 & 8,7 \\
\hline$A L$ & 14,5 & 3,2 & 6,0 & 3,4 & 2,5 & 0,9 & 8,4 & 4,3 \\
\hline SE & 21,5 & 6,4 & 11,4 & 6,4 & 2,7 & 1,4 & 14,1 & 7,8 \\
\hline BA & 12,4 & 2,4 & 8,4 & 4,0 & 1,9 & 0,8 & 10,4 & 4,8 \\
\hline ES & 19,9 & 15,0 & 8,2 & 10,3 & 2,4 & 2,5 & 10,6 & 12,8 \\
\hline MG & 17,3 & 14,9 & 10,4 & 13,5 & 2,3 & 2,6 & 12,6 & 16,1 \\
\hline RJ & 17,7 & 16,4 & 9,1 & 12,6 & 2,3 & 2,9 & 11,3 & 15,5 \\
\hline SP & 13,4 & 13,1 & 12,0 & 11,0 & 2,6 & 2,5 & 15,0 & 13,5 \\
\hline PR & 12,7 & 8,5 & 8,2 & 7,9 & 2,2 & 1,6 & 10,4 & 9,5 \\
\hline SC & 12,5 & 4,6 & 9,9 & 6,6 & 2,2 & 1,4 & 12,1 & 14,6 \\
\hline RS & 9,7 & 9,1 & 8,0 & 7,2 & 3,2 & 2,3 & 11,2 & 9,5 \\
\hline
\end{tabular}

Fonte: SIH/SUS e SIM/MS, 1995 
igualmente concluíram Duchiade \& Andrade. ${ }^{5}$ Os valores semelhantes das taxas de natimortalidade encontrados para o Rio de Janeiro, São Paulo e Rio Grande do Sul, Estados com reconhecida fidedignidade das estatísticas de mortalidade, indicam a viabilidade do SIH/SUS para o cálculo da taxa de natimortalidade hospitalar.

A análise da mortalidade infantil por idade pelo SIH/SUS mostrou uma concentração de óbitos no período neonatal precoce, em semelhança à distribuição etária dos óbitos infantis registrados no SIM/ MS. Adicionalmente, os achados para a mortalidade neonatal, nos Estados das regiões Sudeste e Sul, no mesmo patamar que os estimados pelo SIM/MS, indicaram que a suposição metodológica utilizada (de que os óbitos nos primeiros dias de vida registrados no SIH/SUS correspondem aos nascidos vivos registrados no mesmo sistema) tem coerência.

Dessa forma, os resultados obtidos no presente estudo sugerem que o SIH constitui-se fonte oportuna para o cálculo da taxa de mortalidade neonatal hospitalar, à medida que fornece tanto o numerador quanto o denominador necessários para a sua estimação.

Os valores extremamente baixos em relação ao padrão nacional, encontrados para as taxas de mortalidade neonatal precoce hospitalares nos Estados do Piauí e Maranhão, precisam ainda de investigações específicas. Um possível fator explicativo corresponde à grande freqüência de altas precoces nos hospitais do SUS, na região Nordeste, cujos Estados apresentaram as maiores proporções de gestantes com tempos de permanência inferiores a 24h, conforme estabelecido por análises complementares não apresentadas no presente trabalho. Em outra vertente de explicação, a alta taxa de natimortalidade encontrada no Maranhão faz supor que um excesso de classificações indevidas de óbitos neonatais como natimortos está ocorrendo nesse Estado.

A proporção de óbitos de recém-natos registrados nas "AIH das mães" (cerca de 50\%) mostrou ser resultado merecedor de estudo mais aprofundado. Segundo as normas preconizadas pelo Ministério da Saúde, para emissão de AIH para recém-nascidos, os óbitos registrados nas "AIH da mães" seriam correspondentes, na sua maioria, a crianças sadias. Além disso, a análise dos tempos de permanência das gestantes (dados não apresentados) mostrou que a quase totalidade desses óbitos ocorreu até o terceiro dia de internação da mãe.

Uma das limitações do SIH/SUS decorre da impossibilidade de se distinguir as perdas fetais tardias das precoces. $\mathrm{O}$ mesmo problema ocorre entre as crianças que morreram no período neonatal, já que o peso e a idade gestacional, considerada a variável preditora mais importante da viabilidade fetal, ${ }^{4}$ eram variáveis não incluídas nas AIH até junho de1998, data na qual a Secretaria de Assistência à Saúde do Ministério da Saúde (SAS/MS) incluiu essas variáveis na AIH. ${ }^{11}$ Doravante, será possível realizar análises mais aprofundadas das mortes perinatais.

No que diz respeito à estimativa da taxa de mortalidade neonatal a partir das informações do SIH/SUS, as limitações do método proposto referem-se, primeiramente, à suposição de que o próprio sistema fornece o número de nascidos vivos, podendo-se estar subestimando o denominador da taxa. Sugere-se, assim, que esse procedimento não seja utilizado para o cálculo da mortalidade pós-neonatal, uma vez que são esperadas maiores distorções à medida que o tempo de vida da criança afasta-se do momento do parto. Em segundo lugar, os coeficientes calculados são estimativas de taxas hospitalares, em que os óbitos que ocorreram no domicílio não estão sendo levados em consideração.

No tocante à natimortalidade, erros de classificação dos nascidos vivos como nascidos mortos, não específicos apenas ao sistema SIH/SUS, como citados na literatura, ${ }^{13}$ podem acarretar, por um lado, em estimativas aumentadas das taxas de natimortalidade e, por outro, em subestimativas das taxas de mortalidade neonatal precoce.

De uma maneira geral, a ausência de regras explícitas, de manuais de instrução e/ou literatura, referentes à emissão e ao preenchimento da $\mathrm{AIH}$, acarretam a falta de padronização das informações do SIH/SUS, dificultando as análises comparativas de diferentes regiões do País.

Um outro aspecto a ser considerado é que o procedimento proposto refere-se a estimar apenas taxas hospitalares. Tendo em vista que em municípios onde as informações de registro são precárias e o acesso hospitalar também o é, o cálculo das taxas de mortalidade neonatal seria incorretamente estimado pela metodologia apresentada.

Apesar das limitações apontadas, demonstrou-se que o sistema SIH/SUS é uma fonte alternativa viável para o cálculo da natimortalidade e da mortalidade neonatal hospitalares. Alguns outros aspectos relacionados às informações disponibilizadas pelo SIH/SUS, visando ao desenvolvimento de indicadores da qualidade da assistência, serão explorados em trabalho próximo. Os resultados preliminares sugerem que esse sistema de informações permite aprofundar o estudo da situação das mortes infantis hospitalares vinculadas aos fatores relacionados à assistência ao parto, ainda não sujeitos à investigação prévia no Brasil. 
Considerando a precariedade das estatísticas vitais no Brasil, conclui-se que o uso do SIH/SUS por parte dos pesquisadores de saúde pública, pela agilidade em se obterem as informações, poderá contribuir amplamente ao estudo do comportamento espaço-temporal do componente neonatal da mortalidade infantil no País.

\section{REFERÊNCIAS}

1. Becker RA. Análise da mortalidade: delineamentos básicos. Epidemiológicas. Brasília (DF): Ministério da Saúde; 1991.

2. Beltrão $\mathrm{KI}$, Duchiade MP, Wukhynek PP. Comparação entre alguns métodos para estimação de nascidos vivos. Rio de Janeiro: ENCE; 1990. (Relatórios Técnicos).

3. Camarano AA. Informações demográficas: o que se tem, o que se usa e como se usa? Rev Bras Estud Popul 1990;7:207-17.

4. Chervenak FA, Mccullough LB. The limits of viability. J Perinatol 1997;25:418-20.

5. Duchiade MP, Andrade CLT. Mortes invisíveis: mortalidade perinatal no Estado do Rio de Janeiro, 1979 a 1989. In: Anais do $9^{\circ}$ Encontro Nacional de Estudos Populacionais; 1994; Belo Horizonte. Brasília (DF): ABEP; 1994. vol 1.

6. FIOCRUZ. Assistência médico-sanitária: os serviços de saúde segundo o IBGE. Dados: 1996(20).

7. Giovanella L, Bahia L. Saúde: o poder da atenção pública. Saúde Deb 1995;46.

8. Leal MC, Szwarcwald CL. Evolução da mortalidade neonatal no Estado do Rio de Janeiro, Brasil (1979 a 1993): análise por causa segundo grupo de idade e região de residência. Cad Saúde Pública 1996;12:243-52.

9. Mello Jorge MHP, Gotlieb SLD, Andrade SM. Análise dos registros de nascimentos vivos em localidade urbana no Sul do Brasil. Rev Saúde Pública $1997 ; 31: 78-89$

10. Ministério da Saúde. Secretaria Nacional de Assistência à Saúde. Sistema de Apoio à entrada de dados. Manual das Unidades Hospitalares, Instituto Nacional de Assistência Médica da Previdência Social, SUS, Secretaria Nacional de Assistência à Saúde. Brasília: Ministério da Saúde; 1995.

11. Ministério da Saúde. Secretaria Nacional de Assistência à Saúde. Portaria 2.817. Diário O ficial da União, Brasília (DF): 2 jun 1998

12. Simões CCS, Monteiro CA. Tendência secular e diferenças regionais da mortalidade infantil no Brasil. In: Monteiro CA, organizador. Velhos e novos males da saúde no Brasil. São Paulo: Hucitec; 1996. p. 153-6.

13. Szwarcwald CL, Leal MC, Castilho EA, Andrade CLT. Mortalidade infantil no Brasil: Belíndia ou Bulgária? Cad Saúde Pública 1997;13:503-16.

14. United Nations. Indirect techniques for demographic estimation. New York; 1983. 